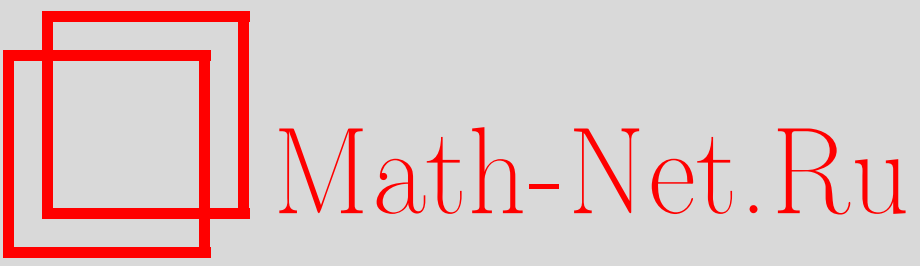

В. А. Ватутин, В. А. Топчий, Основная теорема восстановления для распределений с тяжелыми хвостами, имеющими индекс $\beta \in(0,0.5]$, Теория вероятн. $и$ ее примен., 2013, том 58, выпуск 2, 387-396

DOI: https://doi.org/10.4213/tvp4512

Использование Общероссийского математического портала Math-Net.Ru подразумевает, что вы прочитали и согласны с пользовательским соглашением

http: //www.mathnet.ru/rus/agreement

Параметры загрузки:

IP: 3.95 .254 .165

26 апреля 2023 г., 13:04:20 
7. Бородин А.Н., Салминен П. Справочник по броуновскому движению. Факты и формулы. СПб.: Лань, 2000, 639 с.

Поступила в редакцию

28.II.2012

(c) 2013 г.

ВАТУТИН В. А.*, ТОПЧИЙ В. А.**

\section{ОСНОВНАЯ ТЕОРЕМА ВОССТАНОВЛЕНИЯ ДЛЯ РАСПРЕДЕЛЕНИЙ С ТЯЖЕЛЫМИ ХВОСТАМИ, ИМЕЮЩИМИ ИНДЕКС $\beta \in(0,0.5]^{1)}$}

В работе исследуется асимптотическое поведение приращений функций восстановления, порожденных распределениями, хвосты которых правильно меняются на $\pm \infty$ с показателем $\beta \in(0,0.5]$.

Ключевые слова и фразы: приращения функции восстановления, бесконечное среднее, устойчивые распределения на прямой, нерешетчатое распределение, правильно меняющиеся функции.

1. Введение. Пусть $\xi, \xi_{1}, \ldots, \xi_{n}, \ldots$ - набор независимых случайных величин с одной и той же функцией распределения $G(t)$, и пусть $G^{* k}(t)$ обозначает $k$-кратную свертку $G$ с собой. Если $\mathbf{P}\left(\xi_{i}>0\right)=1$, то соответствующая функция восстановления

$$
U(t):=\sum_{k=0}^{\infty} G^{* k}(t)
$$

корректно определена, т.е. $U(t)<\infty$ для любого $t \in(-\infty, \infty)$. Если же $\mathbf{P}\left(\xi_{i}<0\right)>0$ и случайное блуждание $S_{0}:=0, S_{n}:=\xi_{1}+\cdots+\xi_{n}$ не стремится к бесконечности с вероятностью 1 , то $U(t)=\infty$ для некоторого $t_{0}<\infty$ и, следовательно, для всех $t \leq t_{0}$. В этом случае вместо $U(t)$ естественно для каждого фиксированного $\Delta>0$ изучать величину

$$
\partial U(t)=\partial^{\Delta} U(t):=\sum_{k=0}^{\infty} \partial G^{* k}(t),
$$

где для произвольной функции $F(t)$ мы вводим оператор $\partial=\partial^{\Delta}$ при помощи равенства

$$
\partial F(t)=\partial^{\Delta} F(t):=F(t+\Delta)-F(t) .
$$

Известно, что если $S_{n}$ является невозвратным случайным блужданием, то $\partial U(t)<\infty$ для всех $t$ и $\Delta$. С другой стороны, если блуждание $S_{n}$ возвратно и, скажем, нерешетчато, то $\partial U(t)=\infty$ при всех $t$ и $\Delta>0$ (см. [13, гл. VI, §10]). Заметим, что критерий возвратности блуждания $S_{n}$ для решетчатых распределений приведен в $[17$, гл. II, §8]. В дальнейшем мы будем иметь дело лишь с невозвратными случайными блужданиями.

Имеется много результатов о поведении разностей $\partial U(t)$ для фиксированных значений $\Delta>0$ и $t \rightarrow \pm \infty$ (см., например, [1], [2], [5], [13, гл. ХI]). Случай $|\mathbf{E} \xi| \in(0, \infty)$

* Математический институт им. В.А. Стеклова РАН, Москва, Россия; е-mail: vatutin@mi.ras.ru

** Институт математики им. С. Л. Соболева СО РАН (Омский филиал), Новосибирск, Россия; e-mail: topchij@ofim.oscsbras.ru

1) Работа выполнена при поддержке гранта РФФИ 11-01-00139 и программ РАН «Динамические системы и теория управления» (В. А. Ватутин) и «Развитие методов исследования стохастических моделей, ориентированных на популяционные и биомедицинские приложения» (В. А. Топчий). 
хорошо исследован как для решетчатых, так и для нерешетчатых распределений и носит имя «Основная теорема восстановления». Например, если $\mathbf{E} \xi>0$, то

$$
\lim _{t \rightarrow+\infty} \partial U(t)=\frac{\Delta}{\mathbf{E} \xi}, \quad \lim _{t \rightarrow-\infty} \partial U(t)=0
$$

для нерешетчатых распределений $[13$, гл. XI, §9]. Ситуация становится более сложной, если математическое ожидание случайной величины $\xi$ равно бесконечности или не существует. Наиболее содержательные утверждения в этом случае установлены в предположении, что функция распределения $G(t)$ имеет вид (см. [1])

$$
G(0)=0, \quad 1-G(t)=l(t) t^{-\beta},
$$

где функция $l(t)$ медленно меняется на бесконечности, а $\beta \in(0,1]$, или, более общо, в предположении, что (см. [2]) при $t \geqslant 0$

$$
G(-t)+1-G(t)=l(t) t^{-\beta}
$$

$$
\lim _{t \rightarrow \infty} \frac{G(-t)}{1-G(t)}=\frac{q}{p}
$$

где $p+q=1$ и, как и прежде, функция $l(t)$ медленно меняется на бесконечности, а $\beta \in(0,1]$.

Для дальнейших ссылок мы сформулируем необходимые результаты из работ [1] и [2] в виде следующего утверждения, близкого по виду к теореме 8.6.6 на с. 365 монографии [3].

Положим $m(t):=\int_{0}^{t}(G(-u)+1-G(u)) d u$ при $t>0$ и введем символ $\lim _{t \rightarrow \infty}^{*}$ для обозначения предела $\lim _{t \rightarrow \infty} t \notin E$ по параметру $t$, избегающему некоторое множество $E$ нулевой относительной меры, т.е. избегающему измеримое множество $E$ такое, что $\lim _{x \rightarrow \infty}|E \cap[0, x]| / x=0$. Введем величину $C_{\beta}:=(\Gamma(\beta) \Gamma(2-\beta))^{-1}$. Заметим, что $C_{\beta}=(\sin \pi \beta) /(\pi(1-\beta))$, если $\beta \in(0,1)$.

Теорема 1 (см. [3]). Пусть $G(t)$ - нерешетчатое распределение такое, что $m(t) \rightarrow \infty$ при $t \rightarrow \infty$. Если выполнены условия (2) и (3), то при $\beta \in(0.5,1]$

$$
\begin{aligned}
& \lim _{t \rightarrow+\infty} m(t) \partial U(t)=\Delta \frac{p C_{\beta}}{p^{2}+2 p q \cos (\pi \beta)+q^{2}}, \\
& \lim _{t \rightarrow-\infty} m(t) \partial U(t)=\Delta \frac{q C_{\beta}}{p^{2}+2 p q \cos (\pi \beta)+q^{2}} .
\end{aligned}
$$

Кроме того, если $G(t)$ - нерешетчатое распределение, удовлетворяющее условию (1), то при $\beta \in(0,0.5]$

$$
\liminf _{t \rightarrow \infty} l(t) t^{1-\beta} \partial U(t)=\Delta \frac{\sin \pi \beta}{\pi},
$$

причем для любого $\beta \in(0,1)$

$$
\lim _{t \rightarrow \infty}^{*} m(t) \partial U(t)=\frac{\Delta}{1-\beta} \frac{\sin \pi \beta}{\pi} .
$$

3 а м е ч а н и е. Ясно, что условия (2) и (3) в сочетании с классическими результатами из монографии В. Феллера [13, гл. VIII, §9] влекут

$$
m(t) \sim \frac{1}{1-\beta} l(t) t^{1-\beta}, \quad t \rightarrow \infty,
$$

для $\beta \in(0,1)$ и

$$
m(t) \sim l_{1}(t) \rightarrow \infty, \quad t \rightarrow \infty,
$$

для $\beta=1$, где функция $l_{1}(t)$ медленно меняется на бесконечности, причем так, что $l(t)=o\left(l_{1}(t)\right)($ cм. [7]). 
Утверждение, аналогичное теореме 1 , справедливо и для решетчатых распределений (см., например, [6]). Однако в последнем случае метод доказательства существенно отличается от ситуации с нерешетчатыми распределениями. Стоит напомнить, что имеются примеры, показывающие, что при $\beta \in(0,0.5]$ утверждения вида (4) и (5) не выполняются, вообще говоря, без дополнительных ограничений на функцию распределения $G(t)$. Простой пример такого рода может быть построен при помощи следующих соображений. Если бы соотношения (4) или (5) были всегда справедливы для некоторого $\beta \in(0,0.5]$, то при $|t| \rightarrow \infty$ мы имели бы

$$
\partial G(t)=O(1) \partial U(t)=O(1) \Delta l^{-1}(|t|)|t|^{\beta-1} .
$$

Однако условия (1) и (2) влекут при $|t| \rightarrow \infty$ лишь оценку

$$
\partial G(t)=o(1) \Delta l(|t|)|t|^{-\beta} .
$$

Теперь, в предположении $1-\beta>\beta$, нетрудно построить функцию распределения $G(t)$, для которой $(9)$ противоречит $(8)$. Читатель может найти в работе $[8, \S 5]$ опровергающие примеры такого типа для решетчатого случая.

Следует упомянуть некоторые достаточные условия для справедливости соотношения (5) при $\beta \in(0,1 / 2]$. Например, К. В. Эриксон, основываясь на решетчатых аналогах из работ [4], [5] и [8] и предполагая, что $m(t) \rightarrow \infty$ при $t \rightarrow \infty$, сформулировал в работе [2] следующее утверждение, сопроводив его замечанием, что доказательство этих результатов требует большого объема черновой работы.

Теорема 2. Предположим, что функиия распределения $G(t)$ удовлетворяет условиям (2) и (3) и является абсолютно непрерывной с ограниченной плотностью $g(t)=G^{\prime}(t)$. Допустим далее, что выполнено хотя бы одно из приводимых ниже условий:

i) функиия $g(|t|)=O\left(L(|t|)|t|^{-1-\beta}\right)$ nри всех $t$;

ii) бункиия $g(t)$ монотонна для достаточно больших значений $|t|$;

iii) функиия $g(t)$ абсолютно непрерьвна при $|t| \geq b$, причем $g^{\prime}(t)=$ $O\left(L(|t|)|t|^{-2-\beta}\right)$.

Тогда при выполнении условия і) утверждения (4) и (5) справедливы при $1 / 4<$ $\beta \leq 1$, а при выполнении условия ii) или (u) условия iii) - при всех $\beta \in(0,1]$.

В данной работе мы приводим простое достаточное условие справедливости соотношений (4) и (5) в нерешетчатом случае, которые для $\beta \in(0,1 / 2]$ слабее упомянутых в теореме 2 .

Теорема 3. Пусть выполнены условия (2) и (3) и, кроме того, найдутся положительнье константь $C$ и $T_{0}$ такие, что при $|t| \geq T_{0}$

$$
\partial^{\Delta} G(|t|) \leq \Delta C l(|t|)|t|^{-\beta-1} .
$$

Тогда для любых $\beta \in(0,0.5] u \Delta>0$ справедливы соотношения (4) $и$ (5).

Заметим, что условие (10) выполнено для всех распределений, удовлетворяющих хотя бы одной из гипотез і)-iii), и, более того, не требует абсолютной непрерывности функции $G(t)$. В частности, это подтверждает гипотезу Эриксона из работы [2] о том, что при справедливости предположений (2) и (3) условие і) достаточно для того, чтобы равенство (4) было выполнено при всех $\beta \in(0,1 / 2]$. Мы хотели бы напомнить читателю, что В.А. Топчий доказал в [11] справедливость первоначальной гипотезы Эриксона для распределений с $\beta \in(0,0.5]$, сосредоточенных на положительной полуоси, показав, что условие i) достаточно даже для правильного изменения функции $U^{\prime}(t)$. Он также предложил в [12] более сильную версию условия іiі), обеспечивающую правильное изменение функции $U^{\prime \prime}(t)$. Отметим также, что аналог теоремы 3 для решетчатых распределений, сосредоточенных на положительной полуоси, получил Р. Доней [6]. 
Ясно, что, установив справедливость (5) для любого распределения $G(t)$, удовлетворяющего условию (10), мы тем самым докажем и (4), используя отображение $G(t) \mapsto 1-G(-t)$. В силу этого в дальнейшем мы рассматриваем лишь случай $t>0$ и $t \rightarrow \infty$ и предполагаем, что $p \in(0,1]$.

При доказательстве теоремы 3 мы будем использовать следующие тождества, справедливые при всех $t>0$ и любом $\Delta \geq 0$ :

$$
\begin{aligned}
F_{1} * F_{2}(2 t+\Delta)= & \int_{-\infty}^{t} F_{1}(2 t+\Delta-u) d F_{2}(u)+\int_{-\infty}^{t} F_{2}(2 t+\Delta-u) d F_{1}(u) \\
& +\int_{t}^{t+\Delta} F_{2}(2 t+\Delta-u) d F_{1}(u)-F_{1}(t+\Delta) F_{2}(t)
\end{aligned}
$$

$$
\begin{aligned}
\partial F_{1} * F_{2}(2 t)= & \int_{-\infty}^{t} \partial F_{1}(2 t-u) d F_{2}(u)+\int_{-\infty}^{t} \partial F_{2}(2 t-u) d F_{1}(u) \\
& +\int_{t}^{t+\Delta} F_{2}(2 t+\Delta-u) d F_{1}(u)-F_{2}(t) \partial F_{1}(t) \\
:= & I_{1}+I_{2}+I_{3},
\end{aligned}
$$

где $I_{1}$ и $I_{2}$ обозначают первые два интеграла, а сумма двух последних членов обозначена символом $I_{3}$.

В силу монотонности,

$$
I_{3} \leq\left(F_{2}(t+\Delta)-F_{2}(t)\right)\left(F_{1}(t+\Delta)-F_{1}(t)\right)=\partial F_{1}(t) \partial F_{2}(t) .
$$

В дальнейшем, если не оговорено противное, все соотношения типа

$$
a(t) \sim b(t), \quad a(t)=O(b(t)), \quad a(t)=o(b(t))
$$

будут считаться выполненными при $t \rightarrow \infty$. Например, первое из них означает, что $\lim _{t \rightarrow \infty} a(t) / b(t)=1$. Кроме того, символами $C, C_{1}, C_{2}, \ldots$ будут обозначаться положительные константы, не всегда одинаковые в разных формулах.

Обозначим $L(t)$ функцию, медленно меняющуюся на бесконечности, такую, что

$$
L^{\prime}(t)=o\left(\frac{L(t)}{t}\right), \quad L(t) \sim l(t)
$$

и отношение $L(t) / t^{\beta}$ монотонно убывает при всех достаточно больших значениях $t$. В возможности такого выбора можно убедиться, взяв константу в качестве множителя перед экспонентой в представлении Карамата для медленно меняющихся функций $[13$, гл. VIII, $\S 9$, формула (9.9)], а также обратившись к разделу 5.1 монографии Сенеты [9].

Перепишем условие (10) в эквивалентном виде: существует константа $C_{1}>1$ такая, что при $|t| \geq T_{0}$

$$
\partial G(t) \leq \Delta C_{1} L(|t|)|t|^{-\beta-1}, \quad \beta \in(0,0.5] .
$$

Наше доказательство основывается на следующих двух леммах.

Лемма 1. Существуют константь $H>1 u T_{1}=T_{1}(\beta)>0$ такие, что для $t \geq T_{1}$ u всех $u \in[0,0.5 t]$

$$
0 \leq L(t-u)(t-u)^{-\beta-1}-L(t) t^{-\beta-1} \leq H u L(t) t^{-\beta-2}
$$

$$
\int_{-\infty}^{0.5 t} \partial G(t-u) d G^{* k}(u) \leq \Delta H C_{1} L(t) t^{-\beta-1} .
$$


Д о к а з а т е л ь с т в о. Необходимые утверждения следуют из (2), (13), (14) и свойств правильно меняющихся функций (см., например, [13, гл. VIII, $\S \S 8,9]$ ).

Лемма 2. Пусть выполнены условия (1) и (10) (в виде (14)). Тогда найдутся положительные константы $C_{2}$ u $\varepsilon$ такие, что при $t \geq T=T\left(C_{2}, \varepsilon\right)$ u всех $k \leq$ $\varepsilon t^{\beta} / L(t)$

$$
\partial G^{* k}(t) \leq \Delta k C_{2} L(t) t^{-\beta-1} .
$$

Д о к а з а т е ль с т в о. Используя соотношения (11) и (12), имеем для $k=$ $1,2, \ldots$ :

$$
\begin{aligned}
\partial G^{*(k+1)}(t) \leq & \int_{-\infty}^{0.5 t} \partial G(t-u) d G^{* k}(u)+\int_{-\infty}^{0.5 t} \partial G^{* k}(t-u) d G(u) \\
& +\partial G^{* k}(0.5 t) \partial G(0.5 t):=J_{1}(k)+J_{2}(k)+J_{3}(k) .
\end{aligned}
$$

Из неравенства (14) следует, что при $t \geq T_{2}$

$$
J_{3}(k) \leq \partial G(0.5 t) \leq \Delta 2^{\beta+1} C_{1} L(0.5 t) t^{-\beta-1} \leq 8 \Delta C_{1} L(t) t^{-\beta-1} .
$$

В силу леммы 1 и оценки (17) при $t \geq T_{3}$

$$
J_{1}(k)+J_{3}(k) \leq \Delta C_{1}(H+8) L(t) t^{-\beta-1} .
$$

Зафиксируем теперь число $\varepsilon>0$ такое, что $\varepsilon H<1 / 2$, и положим $C_{2}=2 C_{1}(H+8)$. Будем использовать индукцию для доказательства справедливости неравенства (15) для всех $k$ таких, что $k+1 \leq \varepsilon t^{\beta} / L(t)$.

В силу (14) оценка (15) справедлива при $k=1$.

Допустим, что неравенство (15) верно для некоторого $k \geq 1$ такого, что $k+1 \leq$ $\varepsilon t^{\beta} / L(t)$. Покажем, что оно верно при замене $k$ на $k+1$. Из (2), (3) и теоремы $2[1 \overline{3}$, гл. VIII, § 9] следует, что

$$
\int_{0}^{t} u d G(u)=p(1-\beta) \beta^{-1} L(t) t^{1-\beta}(1+o(1)) .
$$

Выберем величину $T_{1}$ в лемме 1 таким образом, чтобы при $t>T_{1}$ последнюю оценку можно было записать в виде

$$
\int_{0}^{0.5 t} u d G(u) \leq H L(t) t^{1-\beta} .
$$

Принимая во внимание (19), оценки, установленные в лемме 1, и предположение индукции, имеем при всех $t \geq T_{4}$

$$
\begin{aligned}
J_{2}(k) & \leq \Delta k C_{2} \int_{-\infty}^{0.5 t} L(t-u)(t-u)^{-\beta-1} d G(u) \\
& \leq \Delta k C_{2} L(t) t^{-\beta-1}+\Delta k C_{2} L(t) t^{-\beta-2} \int_{0}^{0.5 t} u d G(u) \\
& \leq \Delta k C_{2} L(t) t^{-\beta-1}\left(1+H L(t) t^{-\beta}\right) .
\end{aligned}
$$

Отсюда, в силу $(16),(18)$ и нашего выбора $\varepsilon>0$ и $C_{2}$, получаем

$$
\begin{aligned}
\partial G^{*(k+1)}(t) & \leq \Delta L(t) t^{-\beta-1}\left((k+0.5) C_{2}+(H+8) C_{1}\right) \\
& =\Delta(k+1) C_{2} L(t) t^{-\beta-1},
\end{aligned}
$$

что оправдывает шаг индукции и доказывает лемму 2.

Зададим последовательность $B_{k} \uparrow \infty$ формулой

$$
B_{k}:=\inf \left\{x>0: 1-G(x)+G(-x) \leq k^{-1}\right\} .
$$

В частности,

$$
k\left(1-G\left(B_{k}\right)+G\left(-B_{k}\right)\right)=k B_{k}^{-\beta} l\left(B_{k}\right) \rightarrow 1
$$


при $k \rightarrow \infty$. Последнее при $k \rightarrow \infty$ может быть записано в виде

$$
B_{k} \sim k^{1 / \beta} h(k) \sim k^{1 / \beta} l^{1 / \beta}\left(B_{k}\right)
$$

для некоторой медленно меняющейся функции $h(k)$ (см. $[14,($ В.16)]). Ясно, что

$$
\begin{aligned}
1-G\left(B_{\left[c t^{\beta} / l(t)\right]}\right)+G\left(-B_{\left[c t^{\beta} / l(t)\right]}\right) \sim \frac{l(t)}{c t^{\beta}} \\
\sim \frac{l\left(c^{1 / \beta} t\right)}{\left(c^{1 / \beta} t\right)^{\beta}}=1-G\left(c^{1 / \beta} t\right)+G\left(-c^{1 / \beta} t\right) .
\end{aligned}
$$

Эта эквивалентность и условие (2) ведут к представлениям

$$
B_{\left[(c t)^{\beta} / l(t)\right]} \sim c t \quad \text { и } \quad B_{\left[c t^{\beta} / l(t)\right]} \sim c^{1 / \beta} t
$$

для любого $c>0$.

Используя теорему 3 [13, гл. XVII, §5] и представление (3.18) из [13, гл. XVII, $\S 3]$, нетрудно убедиться в справедливости следующего утверждения.

Лемма 3. Пусть $\xi_{1}, \ldots, \xi_{n}, \ldots$ - последовательность независимьх одинаково распределенных случайных величин с распределением $G(t)$, удовлетворяющим условиям (2) и (3) с $\beta \in(0,1)$. Тогда для любого $t \in(-\infty,+\infty)$

$$
\lim _{n \rightarrow \infty} \mathbf{P}\left(\frac{\xi_{1}+\cdots+\xi_{n}}{B_{n}} \leq t\right)=\int_{-\infty}^{t} g_{\beta}(y) d y,
$$

где плотность $g_{\beta}(t)$ устойчивого распределения с показателем $\beta$ имеет характеристическую функиию

$$
\begin{aligned}
\varphi_{\beta}(z) & :=\int_{-\infty}^{\infty} e^{i z t} g_{\beta}(z) d z \\
& =\exp \left\{-\Gamma(1-\beta)|t|^{\beta}\left(\cos \frac{\pi \beta}{2}-i(p-q) \frac{t}{|t|} \sin \frac{\pi \beta}{2}\right)\right\} .
\end{aligned}
$$

Доказ а т ель с т в о т е о р е мы 3. Согласно локальной теореме Стоуна для нерешетчатых распределений [10],

$$
\partial G^{* k}(t)=\frac{\Delta q_{\beta}\left(t / B_{k}\right)}{B_{k}}+\frac{\delta_{k}}{B_{k}},
$$

где $\delta_{k} \rightarrow 0$ при $k \rightarrow \infty$ равномерно по $t \geq 0$. Оставшаяся часть доказательства является, по существу, повторением рассуждений, использовавшихся в разделе $1^{\circ}$ параграфа 7 работы [1] с единственным отличием: распределение в [1] было сконцентрировано на положительной полуоси, в то время как в нашем случае плотность распределения $G(t)$ является, вообще говоря, положительной в каждой конечной точке действительной прямой. Полагая $n:=\left[A t^{\beta} / l(t)\right]$ и $r:=\left[B t^{\beta} / l(t)\right]$ для $B>A>0$, имеем

$$
t^{1-\beta} l(t) \sum_{k=n}^{r} \partial G^{* k}(t) \sim \Delta \int_{A}^{B} x^{-1 / \beta} q_{\beta}\left(x^{-1 / \beta}\right) d x .
$$

Согласно первой части теоремы 2.2.1 монографии [14] и следствию 2 упомянутой теоремы, $q_{\beta}(0) \in[0, \infty)$ при $\beta<1$, причем функция $q_{\beta}(x)$ непрерывна в нуле. Это означает, что $q_{\beta}\left(t / B_{k}\right) \rightarrow q_{\beta}(0)$ при $k \geq r$ и $t, B \rightarrow \infty$. Принимая во внимание $(23)$, получаем, что для достаточно больших значений $t$ и всех $k \geq r$

$$
\partial G^{* k}(t)<\frac{1+q_{\beta}(0)}{B_{k}} .
$$

В силу теоремы 1 из [13, гл. XVII, §9] и оценок (21) и (22), имеем

$$
\sum_{k=r}^{\infty} B_{k}^{-1} \sim \frac{\beta r^{-1 / \beta+1}}{(1-\beta) h(r)} \sim \frac{\beta r}{(1-\beta) B_{r}} \sim \frac{\beta B t^{\beta}}{(1-\beta) t l(t) B^{1 / \beta}},
$$


или

$$
\sum_{k=r}^{\infty} B_{k}^{-1}=o\left(\frac{t^{\beta-1}}{l(t)}\right)
$$

при $B \rightarrow \infty$.

Применяя лемму 2 для $A<\varepsilon$, мы видим, что

$$
\sum_{k=1}^{n-1} \partial G^{* k}(t) \leq \frac{\Delta L(t)}{t^{\beta+1}} \sum_{k=1}^{n-1} k C_{2}=O(1) \frac{\Delta L(t)}{t^{\beta+1}} n^{2}=A O\left(\frac{t^{\beta-1}}{l(t)}\right)
$$

или

$$
\sum_{k=1}^{n-1} \partial G^{* k}(t)=o\left(\frac{t^{\beta-1}}{l(t)}\right)
$$

при $A \rightarrow+0$. Вспоминая соотношения (24) и (6), заключаем, что

$$
t^{1-\beta} l(t) \partial U(t) \sim t^{1-\beta} l(t) \sum_{k=0}^{\infty} \partial G^{* k}(t) \rightarrow \Delta \int_{0}^{\infty} x^{-1 / \beta} q_{\beta}\left(x^{-1 / \beta}\right) d x:=\Delta I_{\beta},
$$

если вначале $t \rightarrow \infty$, а затем $B \rightarrow \infty$ и $A \rightarrow+0$.

Вычислим теперь значение интеграла $I_{\beta}$ в соотношении (28). Заметим сначала, что по лемме 3

$$
g_{\beta}\left(x^{-1 / \beta}\right)=\frac{1}{\pi} \operatorname{Re} \int_{0}^{\infty} \exp \left\{-\Gamma(1-\beta)|t|^{\beta}\left(\cos \frac{\pi \beta}{2}-i(p-q) \sin \frac{\pi \beta}{2}\right)\right\} e^{-i t x^{-1 / \beta}} d t .
$$

Полагая $u=i t x^{-1 / \beta}$ и рассуждая так же, как и при доказательстве теоремы 2.2 .1 в $[15$, гл. II, $\S 2.2]$ и следствий из нее, получаем

$$
\begin{aligned}
g_{\beta}\left(x^{-1 / \beta}\right)= & \frac{1}{\pi} x^{-1 / \beta} \operatorname{Im} \int_{0}^{\infty} e^{-u} \exp \left\{-x \Gamma(1-\beta)|u|^{\beta} e^{-i \pi \beta / 2}\right. \\
& \left.\times\left(\cos \frac{\pi \beta}{2}-i(p-q) \sin \frac{\pi \beta}{2}\right)\right\} d u \\
= & \frac{1}{\pi} x^{1 / \beta} \operatorname{Im} \int_{0}^{\infty} e^{-u} \exp \left\{-x K_{\beta} u^{\beta}\right\} d u,
\end{aligned}
$$

где

$$
\begin{aligned}
\frac{1}{K_{\beta}} & :=\frac{e^{i \pi \beta / 2}}{\Gamma(1-\beta)(\cos (\pi \beta / 2)-i(p-q) \sin (\pi \beta / 2))} \\
& =\frac{e^{i \pi \beta / 2}(\cos (\pi \beta / 2)+i(p-q) \sin (\pi \beta / 2))}{\Gamma(1-\beta)\left(p^{2}+2 p q \cos (\pi \beta)+q^{2}\right)} .
\end{aligned}
$$

После очевидных преобразований мы видим, что

$$
\begin{aligned}
I_{\beta} & =\frac{1}{\pi} \operatorname{Im} \int_{0}^{\infty} e^{-u} d u \int_{0}^{\infty} \exp \left\{-x K_{\beta} u^{\beta}\right\} d x=\frac{1}{\pi} \operatorname{Im} \frac{1}{K_{\beta}} \int_{0}^{\infty} e^{-u} u^{-\beta} d u \\
& =\frac{1}{\pi\left(p^{2}+2 p q \cos (\pi \beta)+q^{2}\right)} \operatorname{Im} e^{i \pi \beta / 2}\left(\cos \frac{\pi \beta}{2}+i(p-q) \sin \frac{\pi \beta}{2}\right) \\
& =\frac{p}{p^{2}+2 p q \cos (\pi \beta)+q^{2}} \frac{\sin \pi \beta}{\pi} .
\end{aligned}
$$

Это завершает доказательство теоремы 3. 
2. Асимптотики некоторых интегралов. В этом пункте мы докажем некоторые утверждения, связанные с вычислением асимптотического поведения интегралов, полученных при интегрировании некоторых функций относительно функций восстановления, сосредоточенных на положительной полуоси. Мы рассматриваем общую ситуацию и в силу этого вместо функции восстановления $U(t)$ будем использовать положительную монотонную функцию $V(t)$ такую, что при всех $\Delta>0$

$$
\partial V(t)=\Delta t^{\beta-1} l_{V}(t)
$$

где $l_{V}(t)$ - медленно меняющаяся на бесконечности функция. Пусть $l_{P}(t)-$ медленно меняющаяся на бесконечности функция.

Следующее утверждение дополняет теорему 3 работы [1] и теорему 1 из [18] при $\beta \in(0,1 / 2]$.

Теорема 4. Пусть $V(t)$ - монотонная функиия, удовлетворяющая усло-

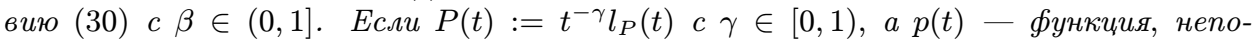
средственно интегрируемая по Риману на $[0, \infty]$, то

$$
\begin{aligned}
\int_{0}^{t} P(t-u) d V(u) & \sim t^{\beta-\gamma} l_{P}(t) l_{V}(t) \int_{0}^{1}(1-u)^{-\gamma} u^{\beta-1} d u, \\
\int_{0}^{t} p(t-u) d V(u) & \sim t^{\beta-1} l_{V}(t) \int_{0}^{\infty} p(u) d u .
\end{aligned}
$$

Eсли $P(t)=t^{-1} l_{P}(t)$, mo

$$
\int_{0}^{t} P(t-u) d V(u) \sim t^{\beta-1} l_{V}(t) \int_{0}^{t} u^{-1} l_{P}(u) d u .
$$

Д о к а з а т е л ь с т в о. Представления (31) и (32) легко следуют из определений правильно меняющихся и непосредственно интегрируемых по Риману функций (см., например, [13, гл. XI, §1]).

Для доказательства соотношения (33) зафиксируем $\varepsilon \in(0,0.5)$ и разобьем промежуток интегрирования на три части: $[0, \varepsilon t),[\varepsilon t,(1-\varepsilon) t)$ и $[(1-\varepsilon) t, t)$. В результате получаем

$$
\int_{0}^{t} P(t-u) d V(u)=\left(\varepsilon^{\beta}+1\right) O\left(t^{\beta-1} l_{V}(t) l_{P}(t)\right)+t^{\beta-1} l_{V}(t)(1+o(1)) \int_{0}^{\varepsilon t} P(u) d u .
$$

Аналогично (7) функция $l_{P I}(t):=\int_{0}^{t} P(u) d u$ является медленно меняющейся и $l_{P}(t)=o\left(l_{P I}(t)\right)$. Эти факты влекут $l_{P I}(\varepsilon t) \sim l_{P I}(t)$ и доказывают $(33)$.

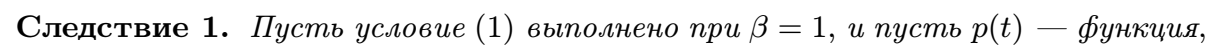
непосредственно интегрируемая по Риману на $[0, \infty]$. Тогда (см. (7))

$$
\int_{0}^{t} p(t-u) d U(u) \sim l_{1}^{-1}(t) \int_{0}^{\infty} p(u) d u
$$

Наш заключительный результат связан с интегралами вида $V * F(t)$, где $F(t)-$ функция распределения.

Теорема 5. Пусть $V(t)$ - функция восстановления, удовлетворяющая условию (30) с $\beta \in(0,1]$, и пусть $F(t)$ - функиия распределения такая, что либо $\partial F(t)=o\left(t^{-1}\right)$, либо

$$
\lim _{\varepsilon \downarrow 0} \limsup _{t \rightarrow \infty} \frac{F(t)-F(t(1-\varepsilon))}{\partial V(t)}=0 .
$$

Тогда при $t \rightarrow \infty$

$$
\partial(V * F)(t) \sim \partial V(t) .
$$


Д о к а $з$ а т е л ь с т в о. Для функции $N=N(t)=o(t)$ имеем

$$
\begin{gathered}
\partial V(t-u) \sim \partial V(t), \quad F(N) \rightarrow 1 \quad \text { при } u \in[0, N] \\
\partial V(t-u)=O(\partial V(t)), \quad 1-F(N)=o(1) \quad \text { при } u \in[N, t(1-\varepsilon)] .
\end{gathered}
$$

Кроме того,

$$
\limsup _{\varepsilon \downarrow 0} \limsup _{t \rightarrow \infty} \frac{1}{\partial V(t)} \int_{t(1-\varepsilon)}^{t} \partial V(t-u) d F(u)=0
$$

$$
\int_{t}^{t+\Delta} V(t+\Delta-u) d F(u) \leq C \partial F(t)=o(\partial V(t)) .
$$

Комбинируя доказанные выше оценки, получаем

$$
\begin{aligned}
\lim _{t \rightarrow \infty} \frac{\partial(V * F)(t)}{\partial V(t)}= & \lim _{t \rightarrow \infty} \frac{1}{\partial V(t)} \int_{0}^{N} \partial V(t-u) d F(u) \\
& +\limsup _{\varepsilon \downarrow 0} \limsup _{t \rightarrow \infty} \frac{1}{\partial V(t)} \int_{N}^{t(1-\varepsilon)} \partial V(t-u) d F(u) \\
& +\limsup _{\varepsilon \downarrow 0} \limsup _{t \rightarrow \infty} \frac{1}{\partial V(t)} \int_{t(1-\varepsilon)}^{t} \partial V(t-u) d F(u) \\
& +\lim _{t \rightarrow \infty} \frac{1}{\partial V(t)} \int_{t}^{t+\Delta} V(t+\Delta-u) d F(u)=1,
\end{aligned}
$$

что и требовалось.

\section{СПИСОК ЛИТЕРАТУРЫ}

1. Erickson K. B. Strong renewal theorems with infinite mean. - Trans. Amer. Math. Society, 1970, v. 151, p. 263-291.

2. Erickson K.B. A renewal theorem for distributions on $\mathbf{R}^{1}$ without expectation. Bull. Amer. Math. Soc., 1971, v. 77, p. 406-410.

3. Bingham N. H., Goldie C. M., Teugels J. L. Regular variation. Cambridge: Cambridge Univ. Press, 1987, 494 p. (Encyclopedia Math. Appl., v. 27.)

4. De Bruijn N. G., Erdös P. On a recursion formula and some Tauberian theorems. J. Res. Natl. Bur. Stand., 1953, v. 50, p. 161-164.

5. Garsia A., Lamperti J. A discrete renewal theorem with infinite mean. - Comment. Math. Helv., 1963, v. 37, p. 221-234.

6. Doney R.A. One-sided local large deviation and renewal theorems in the case of infinite mean. - Probab. Theory Related Fields, 1997, v. 107, p. 451-465.

7. Parameswaran S. Partition functions whose logarithms are slowly oscillating. - Trans. Amer. Math. Soc., 1961, v. 100, p. 217-240.

8. Williamson J. A. Random walks and Riesz kernels. — Pacific J. Math., 1968, v. 25, p. 393-415.

9. Сенета E. Правильно меняющиеся функции. М.: Наука, 1985, 144 с.

10. Stone C.J. A local limit theorem for nonlattice multi-dimensional distribution functions. - Ann. Math. Statist., 1965, v. 36, p. 546-551.

11. Topchii $V$. Renewal measure density for distributions with regularly varying tails of order $\alpha \in(0,1 / 2]$. - Lecture Notes in Statist., 2010, v. 197, p. 109-120.

12. Топчий $B$. Производная плотности восстановления с бесконечным моментом при $\alpha \in(0,1 / 2]$. - Сиб. электрон. матем. изв., 2010, т. 7, с. 340-349.

13. Феллер В. Введение в теорию вероятностей и ее приложения, т. 2. М.: Мир, 1984, $751 \mathrm{c.}$

14. Золотарев В. М. Одномерные устойчивые распределения. М.: Наука, 1983, 304 с.

15. Ибрагимов И. А., Линник Ю. В. Независимые и стационарно связанные величины. М.: Наука, 1965, 524 с. 
16. Фихтенгольи Г. М. Курс дифференциального и интегрального исчисления, т. 2. М.: Наука, 1970, 800 с.

17. Спицер Ф. Принципы случайного блуждания. М.: Мир, 1969, 472 с.

18. Anderson K. K., Athreya K. B. A renewal theorem in the infinite mean case. - Ann. Probab., 1987, v. 15, p. 388-393.

Поступила в редакцию 29.II.2012

\title{
ОБ ОДНОМ ТОЖДЕСТВЕ ДЛЯ РАСПРЕДЕЛЕНИЯ СУММЫ НЕЗАВИСИМЫХ СЛУЧАЙНЫХ ВЕЛИЧИН ${ }^{1)}$
}

\begin{abstract}
В одной из своих статей за 1957 год среди прочего Ф. Спицер опубликовал оценку функции распределения суммы независимых симметричных случайных величин с общим абсолютно непрерывным распределением. Оценка построена с помощью одного тождества для распределения суммы упомянутых случайных величин, доказательство которого не приведено. В настоящей заметке доказано тождество Спицера для любых независимых случайных величин и с его помощью построена оценка функции распределения суммы независимых одинаково распределенных случайных величин. Оценка Спицера получается в качестве частного случая предлагаемой оценки.
\end{abstract}

Ключевые слова и фразы: независимые случайные величины, характеристическая функция, формула обращения.

В статье Ф. Спицера [1] среди прочего доказана лемма (см. следствие в этой заметке). Она нужна Ф. Спицеру для доказательства основного результата в его статье. Эта лемма также представляет самостоятельный интерес. А. Г. Барсегян в своей статье [2] критикует оригинальное доказательство леммы и ставит под сомнение основной результат статьи [1]. В частности автор указывает, что в оригинальном доказательстве используется равенство (равенство (3) в этой заметке), справедливость которого не установлена, и ей кажется, что оно отсутствует в доступной литературе. А. Г. Барсегян предлагает корректные доказательства леммы и равенства Спицера при дополнительном условии на случайные личины. В предлагаемой заметке доказано равенство (1), частным случаем которого является равенство Спицера. В заметке также доказывается оценка (2) функции распределения суммы независимых случайных величин. Оценка Спицера получается из оценки (2) в качестве частного случая.

Пусть на вероятностном пространстве $(\Omega, \mathscr{F}, \mathbf{P})$ определены независимые случайные величины $X_{1}, \ldots, X_{n}$. Обозначим $S_{n}=X_{1}+\cdots+X_{n}$ и $\varphi_{k}(t)=\mathbf{E} \exp \left\{i t X_{k}\right\}$, $t \in \mathbf{R}=(-\infty, \infty), k=1, \ldots, n$, где $i=\sqrt{-1}$.

Теорема 1. Для любого иисла $x>0$ справедливо равенство

$$
\int_{0}^{x} \mathbf{P}\left\{\left|S_{n}\right|<\lambda\right\} d \lambda=\frac{1}{\pi} \int_{-\infty}^{\infty} \frac{1-\cos (x t)}{t^{2}} \prod_{k=1}^{n} \varphi_{k}(t) d t .
$$

* Московский государственный университет им. М. В. Ломоносова, факультет вычислительной математики и кибернетики, Ленинские горы, 119992 Москва, Россия; e-mail: krugvictor@gmail.com

1) Работа выполнена при поддержке РФФИ, грант 11-01-00515-а. 